\title{
TYR03 induces anti-PD-1/PD-L1 therapy resistance by limiting innate immunity and tumoral ferroptosis
}

\author{
Zhou Jiang, ${ }^{1}$ Seung-Oe Lim,, ${ }^{1,2}$ Meisi Yan, ${ }^{1,3}$ Jennifer L. Hsu, ${ }^{1}$ Jun Yao, ${ }^{1}$ Yongkun Wei, ${ }^{1}$ Shih-Shin Chang, ${ }^{1}$ Hirohito Yamaguchi, ${ }^{1,4}$ \\ Heng-Huan Lee, ${ }^{1}$ Baozhen Ke, ${ }^{1}$ Jung-Mao Hsu, ${ }^{1,4}$ Li-Chuan Chan, ${ }^{1}$ Gabriel N. Hortobagyi, ${ }^{5}$ Liuqing Yang, ${ }^{1}$ Chunru Lin, ${ }^{1}$ \\ Dihua Yu, ${ }^{1}$ and Mien-Chie Hung, ${ }^{4,6,7}$
}

'Department of Molecular and Cellular Oncology, The University of Texas MD Anderson Cancer Center, Houston, Texas, USA. ${ }^{2}$ Medicinal Chemistry and Molecular Pharmacology, Purdue University, West Lafayette, Indiana, USA. ${ }^{3}$ Department of Pathology, Harbin Medical University, Harbin, China. ${ }^{4}$ Graduate Institute of Biomedical Sciences, Research Center for Cancer Biology and Center for Molecular Medicine, China Medical University, Taichung, Taiwan. ${ }^{5}$ Department of Breast Medical Oncology, The University of Texas MD Anderson Cancer Center, Houston, Texas, USA. ${ }^{6}$ Department of Biotechnology, Asia University, Taichung, Taiwan. Department of Molecular and Cellular Oncology, The University of Texas MD Anderson Cancer Center, Houston, Texas, USA.

\begin{abstract}
Immune checkpoint blockade therapy has demonstrated promising clinical outcomes for multiple cancer types. However, the emergence of resistance as well as inadequate biomarkers for patient stratification have largely limited the clinical benefits. Here, we showed that tumors with high TYRO3 expression exhibited anti-programmed cell death protein 1/programmed death ligand 1 (anti-PD-1/PD-L1) resistance in a syngeneic mouse model and in patients who received anti-PD-1/PD-L1 therapy. Mechanistically, TYR03 inhibited tumor cell ferroptosis triggered by anti-PD-1/PD-L1 and facilitated the development of a protumor microenvironment by reducing the M1/M2 macrophage ratio, resulting in resistance to anti-PD-1/PD-L1 therapy. Inhibition of TYRO3 promoted tumor ferroptosis and sensitized resistant tumors to anti-PD-1 therapy. Collectively, our findings suggest that TYRO 3 could serve as a predictive biomarker for patient selection and a promising therapeutic target to overcome anti-PD-1/PD-L1 resistance.
\end{abstract}

\section{Introduction}

Immune checkpoint blockade (ICB) therapy that functions by blocking the PD-1/PD-L1 axis has demonstrated long-lasting antitumor activity in multiple cancer types (1-4). The clinical success of anti-programmed cell death protein 1 /programmed death ligand 1 (anti-PD-1/PD-L1) therapy is attributed primarily to the reinvigoration of tumor antigen-specific $\mathrm{T}$ cells inactivated by the engagement of PD-1 on T cells with its ligand PD-L1 on tumor cells (5). However, the response rates are still far from satisfactory, as only approximately $10 \%-30 \%$ of patients could benefit from the therapy as a single agent (1-4), owing to the resistance to anti-PD-1/PD-L1 therapy as well as inadequate biomarkers for patient stratification.

Thus, there is an unmet need to identify effective and specific targets to overcome this resistance. Inhibitors that target RTKs have demonstrated encouraging success against various malignancies (6). Therefore, we sought to identify RTKs that play a role in anti-PD-1/PD-L1 resistance. After RTK candidates are confirmed for their involvement in the resistance, the corresponding therapies to sensitize resistance can be quickly tested, because a large number of inhibitors of RTKs have shown clinical success. Through nonbiased screening assays, we identified TYRO3, a member of the TYRO3, AXL, and MerTK (TAM) RTK family

Conflict of interest: The authors have declared that no conflict of interest exists. Copyright: ( 2021, American Society for Clinical Investigation.

Submitted: April 22, 2020; Accepted: March 3, 2021; Published: April 15, 2021

Reference information: J Clin Invest. 2021;131(8):e139434.

https://doi.org/10.1172/JCl139434. implicated in the activation of numerous downstream pathways including AKT signaling (7), as a potential therapeutic target for overcoming resistance.

Recently, it has been shown that $\mathrm{T}$ cell-induced tumor cell ferroptosis, a nonapoptotic, iron-dependent form of programmed cell death, contributes to $\mathrm{T}$ cell-mediated tumor eradication during immunotherapy (8). Here, we reveal a link between TYRO3 and anti-PD-1/PD-L1 resistance via ferroptosis and show that TYRO3 limited tumor cell ferroptosis and induced anti-PD-1/PD-L1 resistance.

\section{Results}

High TYRO3 expression correlates with a poor prognosis in patients receiving anti-PD-1/PD-L1 therapy. To identify the biomarkers and mechanisms involved in anti-PD-1/PD-L1 resistance, we used a syngeneic mouse tumor model to establish an in vivo model of resistance (Figure 1A). We inoculated 4T1 mammary carcinoma cells into the mammary fat pad (MFP) of BALB/c mice, followed by treatment with mouse anti-PD-1 (anti-mPD-1) antibody, which was previously used for preclinical studies (9). As shown in Figure 1B, parental 4T1 (4T1-P) tumors were responsive to anti-mPD-1 treatment, as evidenced by decreased tumor growth compared with that of tumors treated with IgG. By contrast, resistant $4 \mathrm{~T} 1$ (4T1-R) tumors were nonresponsive to anti-mPD-1, as evidenced by the similar incremental increases in tumor volume observed in tumors treated with IgG and those treated with antimPD-1 antibodies. Next, we screened for kinases that could be involved in anti-PD-1/PD-L1 resistance. To do so, we used a commercially available RTK antibody array system to hybridize the 
A

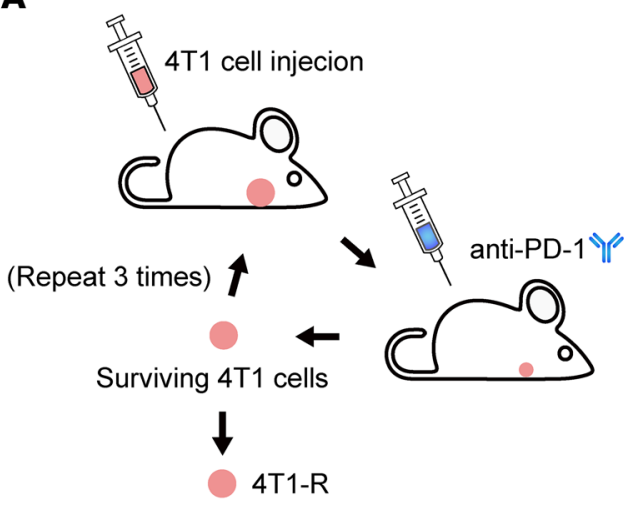

B

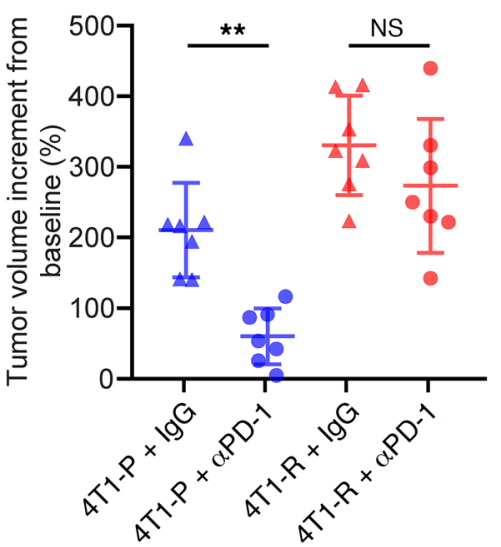

C

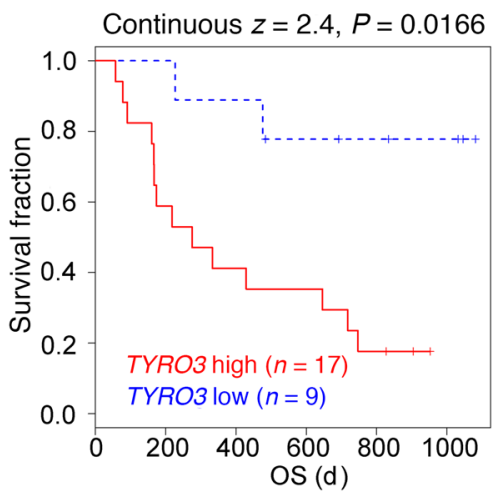

D

Continuous $z=2.76, P=0.00581$

TYRO3 copy high

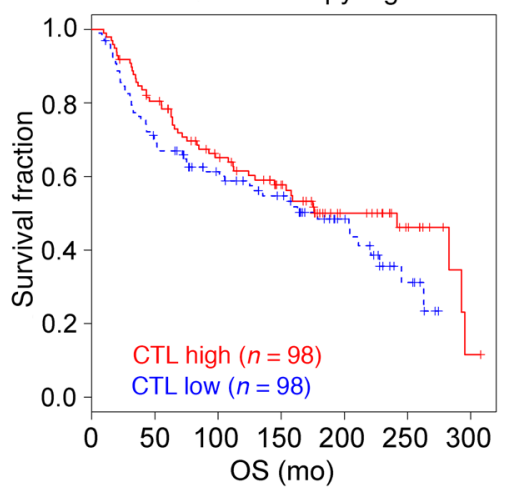

$\mathbf{F}$

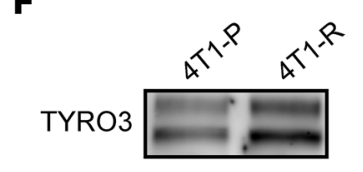

TUBULIN

G

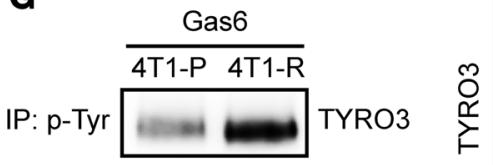
Input $=$ TYRO3
H

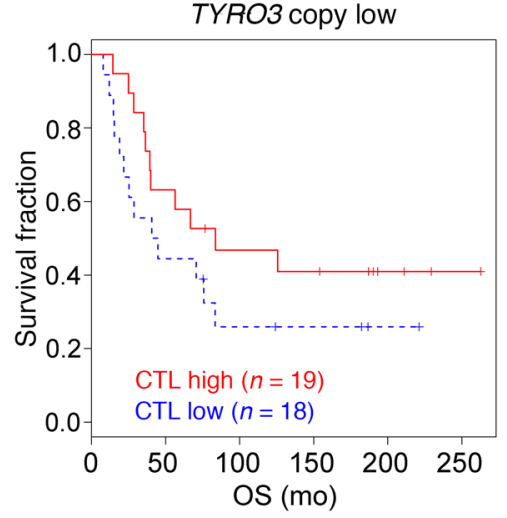

E

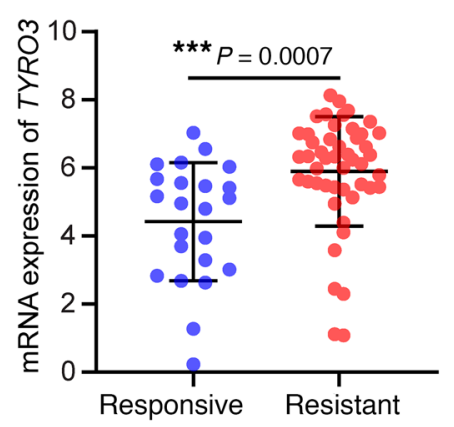

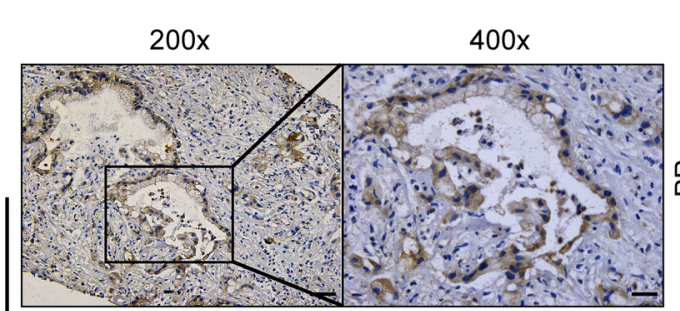
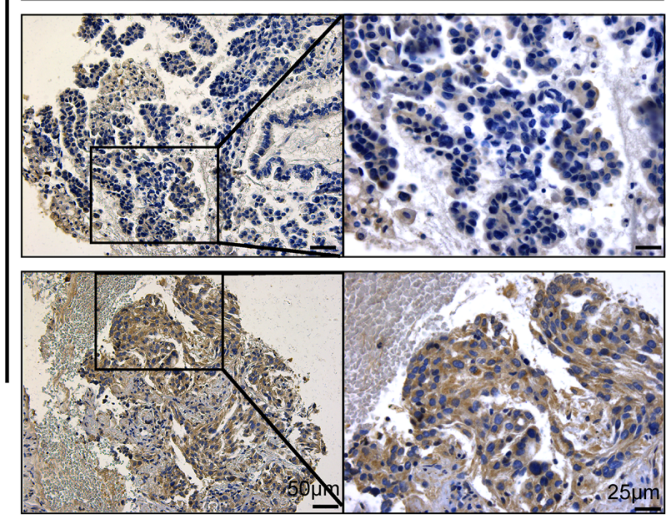

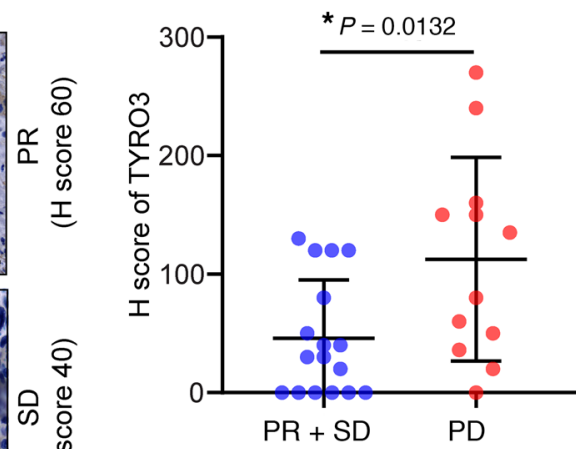

잉

응 
Figure 1. High TYRO3 expression correlates with a poor prognosis for patients receiving anti-PD-1/PD-L1 therapy. (A) Schematic illustrating the establishment of mouse anti-PD-1-resistant cells. (B) Dot plot showing the change in tumor volume compared with baseline in 4T1-P- and 4T1-R tumor-bearing mice treated with anti-PD-1 ( $\alpha$ PD-1) or IgG. $n=7$ for each group. ${ }^{*} P=0.002$ and NS $P=0.44$, by 1 -way ANOVA. (C) Overall survival (OS) of patients with melanoma with high and low TYRO3 mRNA expression who received anti-PD-1 antibody therapy. Continuous $z=2.4 ; P=$ 0.0166 , by 2 -sided Wald test. (D) Relationship between overall survival and CTL levels in patients with breast cancer with high and low TYRO3 gene copy numbers. Continuous $z=2.76 ; P=0.00581$. (E) mRNA expression of TYRO3 in patients with melanoma before and during anti-PD-1 therapy ( $n=23$ responsive patients; $n=48$ resistant patients). ${ }^{* *} P=0.0007$, by 2-tailed, unpaired Student's $t$ test. (F) Western blot analysis of TYRO3 expression in 4T1-P and 4T1-R cells. Tubulin served as a loading control. (C) Immunoprecipitation followed by Western blot analysis of TYRO3 tyrosine phosphorylation levels in 4T1-P and 4T1-R cells in the presence of the TYRO3 ligand Gas6 (100 nM for 30 minutes). TYRO3 served as a loading control. (H) IHC staining of TYRO3 in patients with lung cancer who received anti-PD-1/PD-L1 therapy. H score for TYRO3 expression in patients who were resistant or nonresistant to anti-PD-1/PD-L1 therapy. $n=12$ resistant patients; $n=17$ nonresistant patients. ${ }^{*} P=0.0132$, by 2-tailed, unpaired Student's $t$ test. Scale bars: $50 \mu \mathrm{m}$ (left) and $25 \mu \mathrm{m}$ (right). Data are presented as the mean \pm SD.

array membranes with lysates from 4T1-P or 4T1-R cells and measured the signals using the LI-COR Odyssey Imaging System. The levels of TYRO3, EPHB2, FLT3, and TRKA expression or phosphorylation were higher in 4T1-R cells than in 4T1-P cells, and the increase in TYRO3 was the highest (Supplemental Figure 1A; supplemental material available online with this article; https:// doi.org/10.1172/JCI139434DS1).

To validate the candidates' clinical relevance, we interrogated 2 online databases: Tumor Immune Dysfunction and Exclusion (TIDE), a computational framework developed to evaluate the potential for tumor immune escape using the gene expression profiles of tumor samples (10), and Prediction of Clinical Outcomes from Genomics Profiles (PRECOG), containing nearly 30,000 expression profiles from 166 cancer expression data sets covering a distinct malignant histology (11). We first investigated the correlation between anti-PD-1 resistance and the identified candidates' gene expression profiles from the TIDE database. In the survival data of patients with melanoma treated with antiPD-1 antibodies (12), we found that higher TYRO3 expression levels correlated with shorter overall survival (Figure 1C), suggesting that TYRO3 expression correlates with anti-PD-1 resistance. Expression of the other 3 candidate genes, EPHB2, FLT3, and TRKA, did not correlate with a worse prognosis for patients who received anti-PD-1 therapy (Supplemental Figure 1B). TAM RTKs exhibit structural and functional similarities (13); however, higher AXL or MERTK expression did not correlate with shorter survival times for patients with melanoma who were treated with anti-PD-1 (Supplemental Figure 1C). These findings suggested that TYRO3 is the only TAM RTK member involved in anti-PD-1 resistance. We further explored the clinical relevance of TYRO3 in the PRECOG database. The results showed that higher TYRO3 expression correlated with a worse prognosis for diverse cancer types, including breast cancer, neuroblastoma, bladder cancer, and melanoma, suggesting that TYRO3 is a promising target not only in breast cancer but also in other cancer types (Supplemental
Figure 1D). Thus, among the candidates, we selected TYRO3 for all subsequent analyses.

Next, we investigated the correlation between TYRO3 and T cell antitumor activity in the TIDE database. High infiltration of cytotoxic $\mathrm{T}$ lymphocytes (CTL ${ }^{\text {hi) }}$ into the tumors is known to be correlated with prolonged overall survival of patients (14). Interestingly, we found that this correlation was absent in patients with high TYRO3 expression levels but present in patients with low TYRO3 expression levels (Supplemental Figure 1E). In the tumor microenvironment (TME), TYRO3 is expressed in tumor cells and some other cell types. Considering that our study focused on tumor-expressed TYRO3, the gene copy number was a more accurate reflection of the expression of tumor TYRO3 than of mRNA levels in RNA-Seq data from the patients' tumor bulk. Hence, we examined the correlation of TYRO3 gene copy number and the antitumor activity of cytotoxic $\mathrm{T}$ cells. Echoing the previous result, $\mathrm{CD} 8^{+} \mathrm{T}$ cell levels were not associated with prolonged survival in patients with high TYRO3 gene copy numbers but did mediate prolonged survival in patients with low TYRO3 gene copy numbers (Figure 1D), supporting the notion that TYRO3 reduces the antitumor effects of cytotoxic T cells.

To further determine the correlation between TYRO3 and anti-PD-1 therapeutic outcomes, we analyzed TYRO3 expression in RNA-Seq data from patients with melanoma who received antiPD-1 therapy (12) and found that those with resistant tumors had significantly higher TYRO3 expression levels than did patients whose tumors were responsive (Figure 1E). Western blot analysis also validated the enhanced expression of TYRO3 in the 4T1-R clone (Figure 1F). Given that the status of TYRO3 phosphorylation serves as an indicator of its kinase activity (15), we also measured the levels of phosphorylated TYRO3 (p-TYRO3) in 4T1-R cells. Interestingly, p-TYRO3 was upregulated in 4T1-R cells, which tended to be more responsive to ligand stimulation (Figure 1G). To further determine whether TYRO3 and anti-PD-1/ PD-L1 resistance were commonly correlated in various cancer types, we investigated TYRO3 expression in pre-immunotherapy archived tissues from 29 patients with lung cancer who went on to receive US FDA-approved anti-PD-1/PD-L1 therapy (nivolum$\mathrm{ab}$, pembrolizumab, or atezolizumab) and compared the patients' treatment outcomes. Patients who were resistant to anti-PD-1/ PD-L1 therapy (patients with progressive disease [PD]) had higher levels of TYRO3 expression than did those whose tumor progression was controlled by anti-PD-1/PD-L1 therapy (patients with a partial response [PR] or stable disease [SD]) (Figure 1H). In the resistant cohort, p-TYRO3 levels were also higher (Supplemental Figure 1F). Taken together, these findings suggested that high TYRO3 expression and phosphorylation in patients' tumor tissues correlated with resistance to anti-PD-1/PD-L1 therapy.

TYRO3 renders tumor cells resistant to anti-PD-1 treatment. The above results prompted us to ask whether TYRO3 is sufficient to cause anti-PD-1/PD-L1 resistance. To this end, we compared responses to anti-mPD-1 therapy between Tyro3-overexpressing (Tyro3-OE) and 4T1-P (anti-PD-1-responsive) cells in a syngeneic BALB/c mouse model (Figure 2A, treatment schedule). Tyro3 was overexpressed in anti-PD-1-responsive 4T1-P cells by lentiviral infection, and its expression was validated by immunoblotting (Figure 2B). Anti-mPD-1 treatment significantly reduced tumor 
A

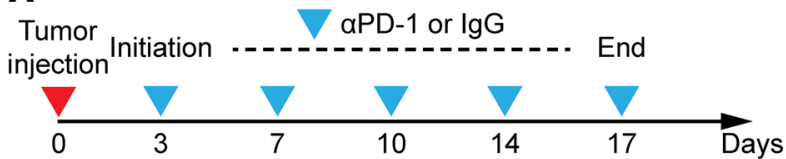

B

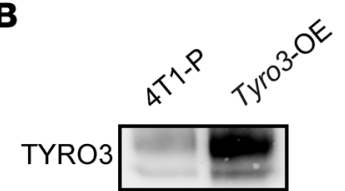

TUBULIN $=-$

E

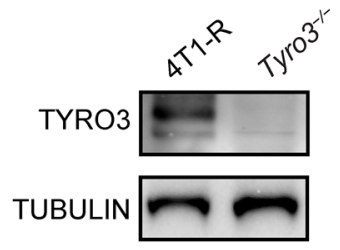

C $\quad-k \cdot 4 \mathrm{~T} 1-\mathrm{P}+\lg \mathrm{G}$

$\widehat{\sigma}^{1200} \rightarrow-4 \mathrm{~T} 1-\mathrm{P}+\alpha \mathrm{PD}-1$

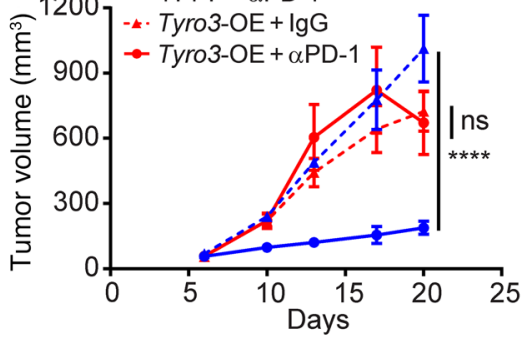

$\mathbf{F}$

-k. $4 \mathrm{~T} 1-\mathrm{R}+\lg \mathrm{g}$

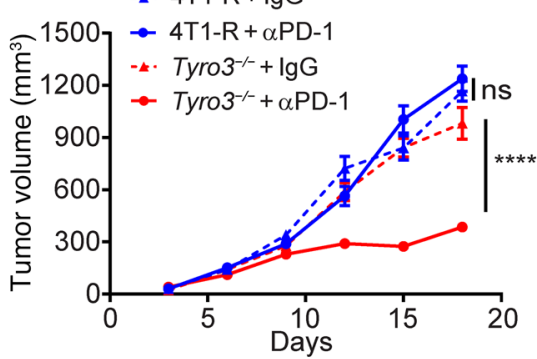

D

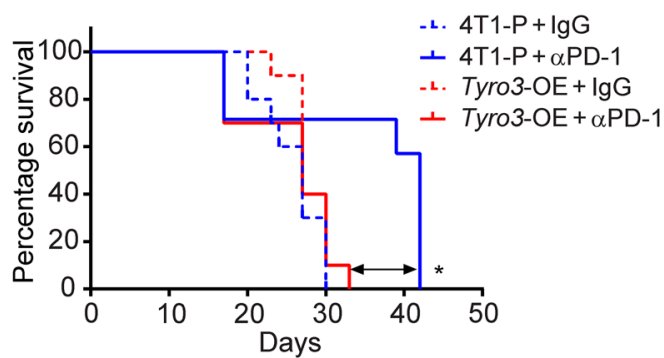

G

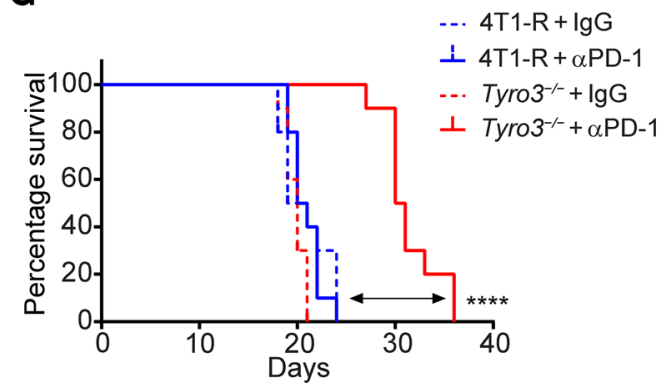

H

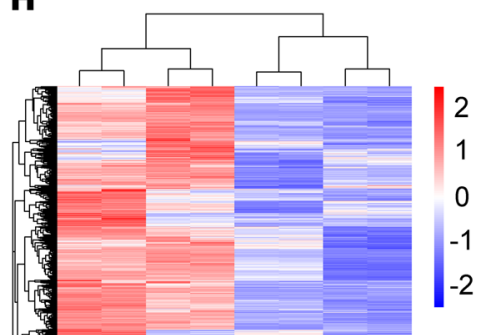

$74.1 \%$

$80.8 \%$

$63.8 \%$

$71.6 \%$

0 Common genes downregulated

Common genes upregulated
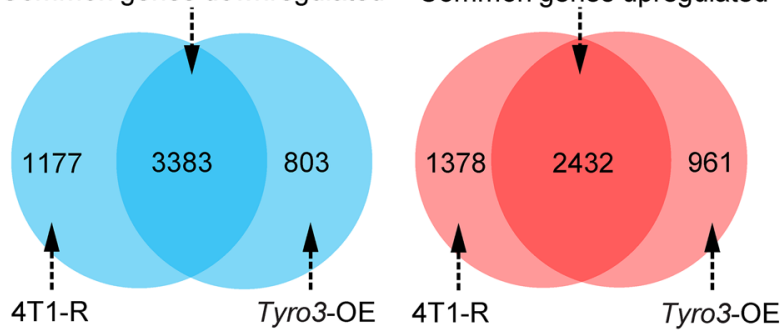

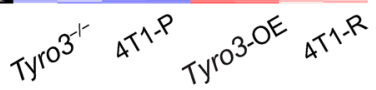

I
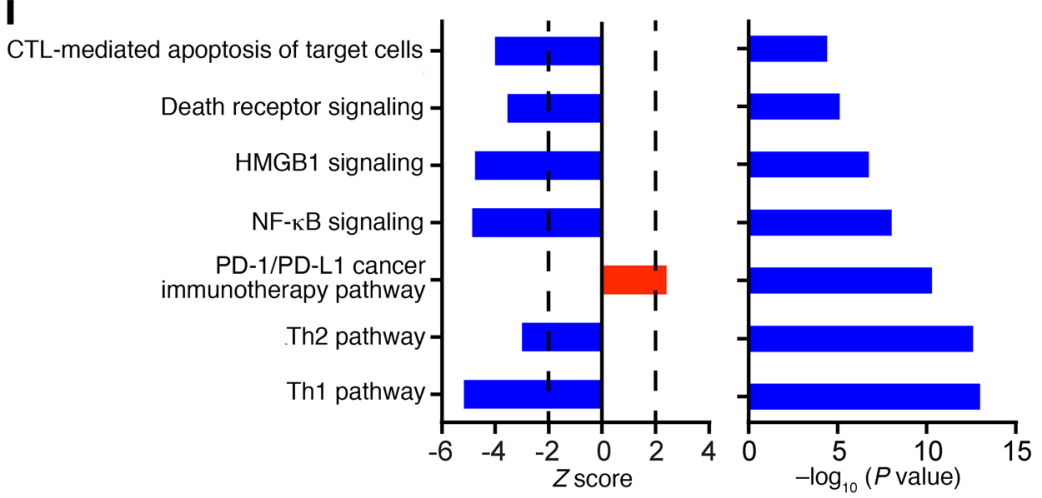
Figure 2. TYRO3 is sufficient to render tumor cell resistant to anti-PD-1 treatment. (A) Mice were given anti-mPD-1 antibody starting on the third day after tumor inoculation and treated on the indicated days for a total of 5 treatments. (B) TYRO3 expression in 4T1-P and Tyro3-OE cells. Tubulin was used as a loading control. (C) Tumor growth in mice bearing 4T1-P and Tyro3-OE tumors. Mice were given anti-IgG or anti-mPD-1 antibody. $n=10$ mice/group. ${ }^{* * *} P<0.0001$ and NS $P=0.98$, by 2 -way ANOVA. Data are presented as the mean \pm SEM. (D) Survival of mice in the 4T1-P and Tyro3OE groups. $n=10$ mice per group. ${ }^{*} P=0.0158$, by 2 -sided log-rank test. (E) Western blot analysis of TYRO3 expression in 4T1-R and Tyro3 $\%$ cells. Tubulin was used as a loading control. (F) Tumor growth in mice bearing 4T1-R and $\mathrm{Tyro3}^{--}$tumors. The mice were given anti-lgG or anti-mPD-1 antibody treatment. $n=10$ mice per group. ${ }^{* * *} P<0.0001$ and NS $P=$ 0.65 , by 2-way ANOVA. Error bars are present but nominal in some cases. Data are presented as the mean \pm SEM. (C) Survival of mice in the 4T1-R and Tyro $^{-/}$groups ( $n=10$ mice per group). ${ }^{* * * *} P<0.0001$, by 2 -sided log-rank test. (H) Hierarchical clustering analysis was performed with the $\log _{10}$ (FPKM+1) of union differentially expressed genes in all comparison groups (4T1-P, Tyro3-OE, 4T1-R, and $\mathrm{Tyro3}^{-/}$), with the mean value used for each group. Venn diagram highlighting the similarities and differences in significantly downregulated (left) and upregulated (right) genes in 4T1-R and Tyro3-OE cells. (I) IPA of genes correlated with TYRO3 in 287 patients with melanoma. The $z$ score was calculated by Spearman's correlation and $P$ values using IPA.

growth (Figure 2C) and extended survival (Figure 2D) in mice bearing 4T1-P tumors but not in those with Tyro3-OE tumors. These results indicated that, although 4T1-P tumors responded to anti-mPD-1 treatment, overexpression of Tyro3 promoted resistance in those tumors.

Next, to further determine whether TYRO3 is required for anti-PD-1/PD-L1 resistance in 4T1-R-resistant cells, we knocked out Tyro3 in 4T1-R cells (Tyro3--) by CRISPR/Cas9 (Figure $2 \mathrm{E})$. We compared the response to anti-mPD-1 between 4T1-R tumors and Tyro $3^{--}$tumors under the same treatment schedule described above and shown in Figure 2A. Remarkably, mice bearing Tyro3 $3^{--}$, but not 4T1-R, tumors were sensitive to anti$\mathrm{mPD}-1$ treatment, as evidenced by the significant reduction in tumor growth (Figure 2F). Consistently, mice bearing $\mathrm{Tyro3}^{-1-}$ tumors treated with anti-mPD-1 survived longer than did those with 4T1-R tumors (Figure 2G). We further showed by cell counting (Supplemental Figure 2A) and a CFSE cell proliferation assay (Supplemental Figure 2B) that neither Tyro3 overexpression nor Tyro3 depletion status affected 4T1 cell growth (Supplemental Figure 2A). These results demonstrated a vital role of TYRO3 in anti-PD-1/PD-L1 resistance.

To better understand the mechanistic role of TYRO3 in antiPD-1/PD-L1 resistance, RNA extracted from 4T1-P, Tyro3-OE, 4T1-R, and $\mathrm{Tyro3}^{-/-}$cells were subjected to whole-transcriptome analysis. The transcriptomic changes observed were highly similar between the 2 resistant cell lines Tyro3-OE and 4T1-R, with a high percentage of overlap in these changes (Figure $2 \mathrm{H}$ ), supporting the role of TYRO3 in promoting tumor cell resistance to antiPD-1/PD-L1 therapy. The correlation between TYRO3 and antiPD-1/PD-L1 resistance was also supported by Ingenuity Pathway Analysis (IPA) of genes coexpressed with TYRO3 in patients with melanoma from The Cancer Genome Atlas (TCGA) database (ref. 16, Figure 2I, and Supplemental Table 1), showing that TYRO3 expression was positively correlated with the PD-1/PD-L1 cancer immunotherapy pathway $(z$ score $=2.405)$. Consistent with the results shown in Figure 1D and Supplemental Figure 1E, we found that pathways related to $\mathrm{T}$ cell-mediated antitumor responses, such as CTL-mediated apoptosis and death receptor signaling, were negatively correlated with TYRO3 $(z$ score $=-4$ and -3.53 , respectively). Inflammatory response-related pathways, such as Th1 activation $(z$ score $=-5.1)$, Th2 activation $(z$ score $=-2.9)$, and NF- $\mathrm{KB}$ signaling $(z$ score $=-4.8)$, were negatively correlated with TYRO3 expression, which is in line with previous findings that low inflammatory response levels induce a protumorigenic TME and contribute to ICB therapy resistance (17). HMGB1 is a damage-associated molecular pattern (DAMP) molecule and is released by ferroptotic cells (18), and we found that HMGB1 signaling was negatively correlated with TYRO3 expression $(z$ score $=-4.75)$. Together, these data point to the role of TYRO3 in anti-PD-1/PD-L1 resistance and suggest that TYRO3 favors an antiinflammatory TME.

TYRO3 induces anti-PD-1/PD-L1 resistance by suppressing ferroptosis and favoring a protumor TME. To interrogate the functions of TYRO3 in the TME, we first evaluated the overall changes in immunological profiles between Tyro3-OE 4T1 and 4T1-P tumors. The tumors were disassociated and subjected to immune cell profiling by time-of-flight mass cytometry (CyTOF) followed by PhenoGraph clustering analysis (Figure 3, A and B, Supplemental Figure 3A, and ref. 19). We showed that in $\mathrm{CD}^{-} 5^{-}$tumor cells, Tyro3 overexpression was not associated with PD-L1 or caspase-3 (Supplemental Figure 3, B and C). We observed decreased levels of M1-like macrophages, which are proinflammatory and have antitumor activity, in Tyro3-OE tumors compared with 4T1-P tumors. The decreased ratio of M1/M2 in Tyro3-OE tumors suggested that tumor-expressed TYRO3 promotes M1-to-M2 macrophage polarization. Moreover, although TYRO3 reduced the antitumor effects of cytotoxic T cells in patients (Figure 1D and Supplemental Figure 1E), there was no significant difference in the levels of $\mathrm{CD} 8^{+} \mathrm{T}$ cells or Tregs between 4T1-P and Tyro3-OE tumors. This discrepancy indicated that the presence of tumor cell-intrinsic mechanisms caused the resistance to $\mathrm{T}$ cell cytotoxicity.

We further validated the antiinflammatory function of TYRO3 in vitro by culturing THP1 monocytes or bone marrow-derived macrophages (BMDMs) with the conditioned medium (CM) from Tyro3-OE BT549 cells or Tyro3-OE and Tyro3 $\% 4$ T1 tumor cells. In THP1 cells, we assessed M1/M2 polarization by measuring mRNA expression of the classical M1 markers, proinflammatory cytokines IL6 and CXCL1O, and major histocompatibility complex class II (antigen-presenting molecule HLADRA1), as well as the M2 markers CD206 (also known as MRC1), arginase 1 (ARG1), and the antiinflammatory cytokine IL1O. CM from Tyro3-OE BT549 cells decreased the levels of the M1 marker HLADRA1 and increased the M2 markers MRC1, ARG1, and IL1O in THP1 cells (Supplemental Figure 3D). In addition, we analyzed the M1 marker CD11c and the M2 marker CD206, respectively, in BMDMs that were cultured in CM from Tyro3-OE or Tyro3 $-14 \mathrm{~T} 1$ cells. CM from Tyro3-OE 4T1 cells decreased CD11c and increased CD206 levels in BMDMs, whereas CM from 4T1-Tyro3-- cells decreased CD206 levels in BMDMs, indicating that TYRO3 promotes macrophage polarization from M1 to M2 (Supplemental Figure 3, $\mathrm{E}$ and F). Because the supernatant from tumor cells promoted macrophage polarity, tumor-secreted proteins, including spe- 
A

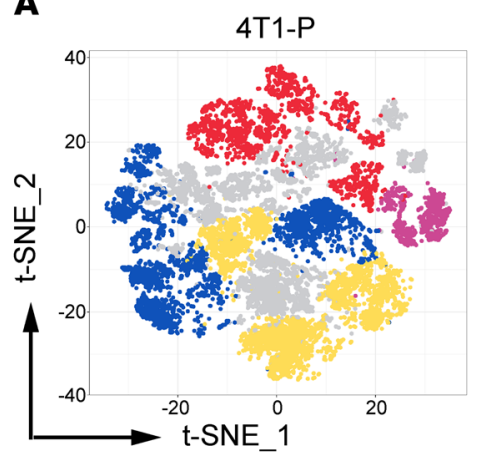

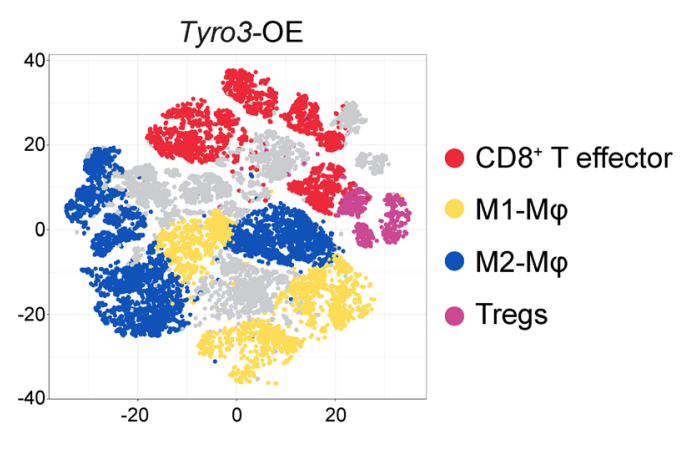

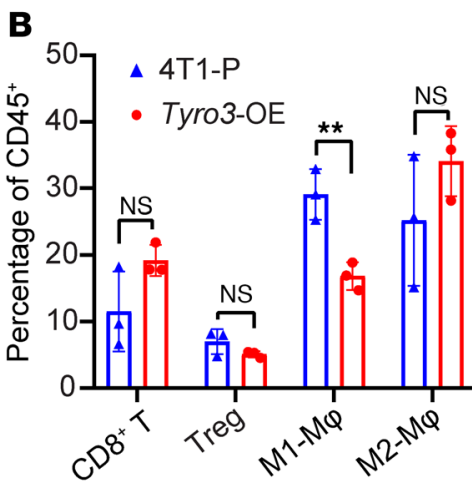

Figure 3. TYRO3 favors a protumor TME. (A) $t$-distributed stochastic neighbor embedding ( $t$-SNE) plot of tumor-infiltrating leukocytes overlaid with color-coded clusters. Five thousand cells are displayed in each $t$-SNE plot. (B) Frequency of clusters of the indicated immune cell subsets. Data represent the mean $\pm \mathrm{SD}$ ( $n=3$ mice per group). CD8 ${ }^{+} \mathrm{T}$ cells, $P=0.11$; Tregs, $P=0.16$; M1 macrophages (M1-M $\varphi$ ), ${ }^{* *} P=0.008$; and $\mathrm{M} 2$ macrophages $(\mathrm{M} 2-\mathrm{M} \varphi$ ), $P=0.24$, by 2-tailed, unpaired Student's $t$ test. Clusters 13, 20, 24, and 29 comprised CD3 ${ }^{+}$CD8 $8^{+}$cytotoxic T cells; clusters 3, 14, and 16 comprised CD3 $3^{+}$CD4 ${ }^{+}$CD25 ${ }^{+}$Tregs; clusters $1,2,4,5,7$, and 12 comprised CD11b+CD68+CD206-CD80+ M1-like macrophages; and clusters 8, 15, 17, 23, 26, 28, and 30 comprised CD11b+CD68+CD206 ${ }^{+}$arginase $1^{+}$M2-like macrophages.

cific cytokines, chemokines, and growth factors were therefore the candidates that potentially contributed to TYRO3-mediated M1-to-M2 polarization. Inhibition of VEGF signaling is known to contribute to M2-to-M1 macrophage polarization (20). Compared with 4T1-P cells, RNA-Seq revealed that VEGF expression was upregulated in the resistant cell lines 4T1-R and Tyro3-OE. The upregulation of VEGF was validated in both Tyro3-OE 4T1$\mathrm{P}$ and TYRO3-OE BT549 tumor cells (Supplemental Figure 3, G and $\mathrm{H}$ ). Thus, VEGF could be the intermediary between TYRO3 and macrophage polarization. Thus, we applied an inhibitor of VEGF/VEGFR signaling, axitinib, to test this possibility. As shown in Supplemental Figure 3I, CM from TYRO3-OE BT549 cells significantly decreased the expression of M1 markers and increased the expression of M2 markers, which was consistent with the data shown in Supplemental Figure 3D. As expected, addition of the VEGFR inhibitor entirely abolished the TYRO3-mediated effects on macrophage polarization. These results collectively indicated that TYRO3 decreased the M1/M2 ratio by upregulating VEGF, thereby promoting a protumor TME.

To better understand TYRO3-associated resistance mechanisms, we looked further into tumor cell-intrinsic resistance. A recent study revealed a novel mechanism by which $\mathrm{T}$ cells eliminate tumor cells by inducing tumor cell ferroptosis (8). We speculated that tumor cells could inhibit ferroptosis to resist the antitumor activity of T cells. Furthermore, because HMGB1 can be released by ferroptotic cells (18) and the HMGB1 signaling pathway is negatively associated with TYRO3 mRNA expression (Figure 2I), we postulated that TYRO3-mediated resistance could have been achieved by the suppression of tumor cell ferroptosis. To this end, we first examined relative changes in the expression of genes related to ferroptosis $(21,22)$. These changes in expression levels indicated that TYRO3 could be involved in regulating ferroptosis: in Tyro3-OE 4T1 tumor cells, genes that block ferroptosis were upregulated (Slc4Oa1, Slc7a11, Slc3a2, Gpx4, Fth1, and Blvrb), whereas genes that enhance ferroptosis were downregulated (Slc5a1, Tfrc) compared with levels in 4T1-P cells (Figure 4A and Supplemental Figure 4A). This observation prompted us to determine the role of TYRO3 in the inhibition of ferroptosis. Of note, in the database of patients with melanoma who received anti-PD-1 therapy, we validated that SLC3A2, a system $\mathrm{X}_{\mathrm{c}}^{-}$cysteine/glutamate antiporter component that prevents lipid peroxidation and ferroptosis (23), was significantly coexpressed with TYRO3 (Figure $4 \mathrm{~B}$ ). We further confirmed the positive correlation between SLC3A2 and TYRO3 in patients with breast cancer in TCGA (Supplemental Figure $4 \mathrm{~B}$ ) and patients with melanoma in a tissue microarray (Supplemental Figure 4C). Collectively, these results raised the possibility that TYRO3 upregulates genes that protect tumor cells from undergoing immunotherapy-induced ferroptosis.

To test this possibility, we treated 4T1-P or Tyro3-OE $4 \mathrm{~T} 1$ tumor-bearing mice with anti-PD-1 and analyzed the effects of treatment on lipid peroxidation, a functional marker of ferroptosis. With anti-PD-1 treatment, Tyro3-OE CD45- tumor cells displayed lower lipid ROS levels than did 4T1-P CD45- tumor cells, whereas the addition of anti-PD-1 significantly increased lipid ROS in 4T1-P, but not Tyro3-OE, tumor cells (Figure 4C), supporting the notion that TYRO3 suppresses anti-PD-1-induced tumor cell ferroptosis. According to Wang et al., tumor ferroptosis is induced by $\mathrm{T}$ cell-secreted IFN- $\gamma$; however, there was no significant change in the $\mathrm{CD} 8^{+} \mathrm{T}$ cell frequency or activity, with IFN- $\gamma$ and granzyme $\mathrm{B}$ as activity indicators, between anti-PD-1-treated Tyro3-OE 4T1 tumors and 4T1-P tumors (Figure 3B, Figure 4D, and Supplemental Figure 4D). Thus, the decrease in ferroptosis could be regulated by mechanisms intrinsic to tumor cells.

To validate this hypothesis, we treated 4T1-P and Tyro3-OE 4T1 cells with the ferroptosis inducer erastin and the ferroptosis inhibitor ferrostatin 1 (Fer-1) in vitro. Compared with parental cells, Tyro3 overexpression suppressed erastin-induced cell death as measured by $7-\mathrm{AAD}^{+}$cells (Figure $4 \mathrm{E}$ ). Consistent with the previous observations (8), the changes in lipid peroxidation were not as obvious as the changes in cell death. Tyro 3 overexpression inhibited erastin-induced lipid ROS (Figure 4F), while the Tyro3 overexpression-mediated decrease in cell death and lipid ROS was absent when Fer-1 blocked ferroptosis. We further confirmed the decreased lipid peroxidation in Tyro3-OE tumor cells using a malondialdehyde (MDA) assay (Supplemental Figure 4E). Like- 
wise, we found that Tyro3 depletion increased erastin-induced cell death (Figure 4G) and lipid peroxidation (Figure 4H) and that Fer-1 eliminated these effects. The increased lipid peroxidation in $\mathrm{Tyro3}^{-/-}$tumor cells was also confirmed by the MDA assay (Supplemental Figure $4 \mathrm{~F}$ ). Together, these results indicated that TYRO3 is essential for protecting tumor cells from ferroptosis.

NRF2, a key mediator in blocking ferroptosis $(24,25)$, activates the transcription of ferroptosis-inhibitory genes, including $4 \mathrm{~F} 2$ cell-surface antigen heavy chain (SLC3A2), cystine/glutamate transporter (SLC7A11), ferritin light chain ( $F T L)$, ferritin heavy chain (FTH1), glutathione peroxidase 4 (GPX4), ferroportin (SLC4OA1), and biliverdin reductase A/B (BLVRA/B) (22). Most of these genes were also shown to be upregulated by TYRO3 (Figure 4A). Thus, we hypothesized that NRF2 is involved in TYRO3mediated ferroptosis suppression. To validate this hypothesis, we used a dual-luciferase reporter assay to show that NRF2 transcriptional activity was increased in $293 \mathrm{~T}$ cells transfected with a TYRO3-overexpressing plasmid compared with 293T cells without TYRO3 overexpression (Figure 4I and Supplemental Figure $4 G)$. The PI3K/AKT signaling pathway, which is downstream of TAM kinases, can increase NRF2 transcriptional activity (26). We showed that TYRO3-OE-mediated NRF2 transcriptional activation was abolished by the AKT inhibitor MK2206 (Figure 4I) or by a dominant-negative mutant AKT plasmid (Supplemental Figure $4 \mathrm{G}$ ). Moreover, the TYRO3-mediated reduction of lipid peroxidation was no longer observed in the presence of MK2206 (Figure $4 \mathrm{~J})$. These results suggested that TYRO3 inhibits ferroptosis via the AKT/NRF2 axis.

The clearance of dying cells by phagocytes depends on recognition of the "eat-me signals" exposed by apoptotic cells. Phosphatidylserine (PS) is a well-defined and key "eat-me" molecule that binds to bridging molecules, such as the TAM kinase ligands protein S (Pros1) and Gas6. The presence of the PS-Pros1-Gas6 complex on the surface of dying cells facilitates phagocytosis by activating phagocytes expressing MerTK (13). Although Pros1 activates both TYRO3 and MerTK when binding to PS, it specifically activates TYRO3 without PS binding (27). On the basis of the above results (Figure 4, E-H), it is worth determining whether the Pros1 "eat-me" signal delivered by apoptotic cells could activate tumor cell-expressed Pros1-receptor TYRO3 to promote tumor cell survival via the suppression of ferroptosis. To this end, we assessed the effects of Pros1-delivered signaling without PS binding in the presence or absence of erastin. As expected, Pros1 treatment of 4T1 and PY8119 tumor cells inhibited erastin-induced lipid peroxidation (Figure $4 \mathrm{~K}$ and Supplemental Figure $4 \mathrm{H}$ ). This effect no longer existed in the absence of TYRO3, as shown in Figure 4L, supporting the notion that the Pros1 "eat-me" signal from apoptotic cells could inhibit tumor cell ferroptosis through TYRO3 activation. Taken together, these findings suggested that TYRO3 inhibits tumor cell ferroptosis and supports a protumor TME by reducing the M1/M2 ratio. In addition, tumor cells may utilize the Pros1 "eat-me" signal from adjacent dying cells to promote the survival of tumor cells by activating TYRO3 to suppress ferroptosis.

Inhibition of TYRO3 enhances ferroptosis and sensitizes resistant tumors to anti-mPD-1 therapy. The potential role of TYRO3 in triggering resistance provided us a strong rationale to further apply TYRO3 inhibition to sensitize resistant tumors to anti-PD-1 ther- apy. To this end, we treated 4T1-R cells with LDC1267, a TYRO3 inhibitor with an $\mathrm{IC}_{50}$ of $8 \mathrm{nM}, 29 \mathrm{nM}$, and $5 \mathrm{nM}$ for TYRO3, AXL, and MerTK, respectively (28), and found that it effectively decreased TYRO3 phosphorylation (Figure 5A). Compared with the vehicle control, LDC1267 treatment increased tumor cell death and lipid peroxidation in 4T1-R cells, while the effects were absent in Tyro3 ${ }^{-/-}$cells (Figure 5, B and C).The increased lipid peroxidation by LDC1267 treatment in 4T1-R cells was further validated by MDA assay (Supplemental Figure 4I). We also observed increased lipid peroxidation and tumor cell death by LDC1267 in B16F10 and PY8119 cells, and the effects were absent in Tyro3-cells (Supplemental Figure 4, J-O). These findings suggested that inhibition of TYRO3 enhances tumor cell ferroptosis.

We further investigated TYRO3 inhibition in the anti-PD-1 resistance mouse model. Mice bearing 4T1-R tumors were intraperitoneally injected with anti-mPD-1, LDC1267, or their combination (Figure 5D). The combination treatment significantly reduced tumor growth (Figure 5E) and prolonged mouse survival (Figure 5F) compared with the control or any single agent. Furthermore, the combination treatment was well tolerated in the animals, as evidence by the biochemical indicators of kidney function (blood urea nitrogen [BUN]) and liver function (aspartate aminotransferase [AST] and alanine aminotransferase [ALT]), which were well within their normal range (Figure 5, G-I). Collectively, these results indicated that targeting TYRO3 in combination with anti-PD-1 has the potential to overcome anti-PD-1/PD-L1 resistance with relatively low levels of toxicity.

Given our findings, we proposed a model in which high expression of TYRO3 or TYRO3 activation by its ligands on apoptotic cells triggers the downstream AKT/NRF2 pathway, followed by the transcription of genes that inhibit ferroptosis (Figure 5J). In the current study, we demonstrated that Tyro3 overexpression inhibited tumor cell ferroptosis (Figure 4, C, and E-H) and reduced the ratio of M1/M2 macrophages (Figure 3B). Hence, we proposed that TYRO3 suppresses tumor cell ferroptosis, favors a protumor TME by promoting M1-to-M2 polarization, and consequently facilitates tumor survival during anti-PD-1 therapy.

\section{Discussion}

The present study showed a significant clinical correlation between TYRO3 and anti-PD-1/PD-L1 therapy resistance in patients with cancer, suggesting the utility of TYRO3 as a predictive biomarker for patient stratification to improve therapeutic outcomes. Mechanistically, our results demonstrated that TYRO3 suppressed tumor cell ferroptosis and contributed to a protumor TME, thereby promoting resistance to anti-PD-1/PD-L1. Intriguingly, our study pointed out a potential function of the "eat-me" signal in adjacent apoptotic cells that can be used by TYRO3expressing tumor cells to promote their survival by suppressing ferroptosis. Furthermore, we demonstrated that a TYRO3 inhibitor could reverse TYRO3-mediated protumor effects and sensitize resistant tumors to anti-PD-1 therapy, which supported TYRO3 as a promising therapeutic target.

In this study, we established the resistance model in $4 \mathrm{~T} 1$ cells, a murine triple-negative breast cancer (TNBC) cell line. TNBC is an aggressive breast cancer subtype: more than half of the patients do not respond to front-line therapy (29), and less than $20 \%$ 
A
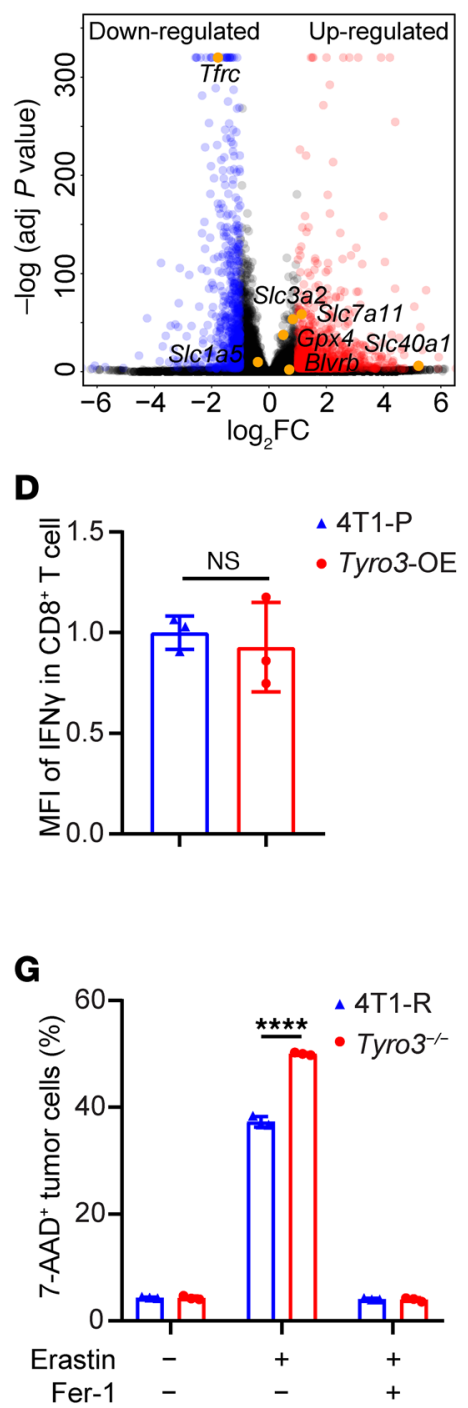

J

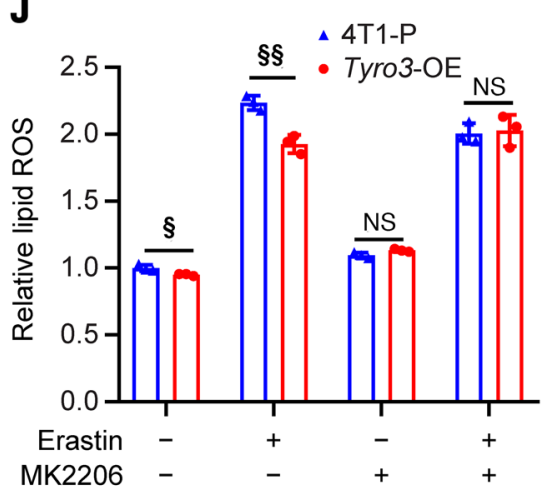

B

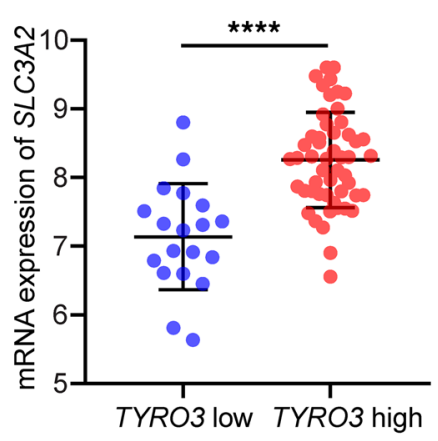

E

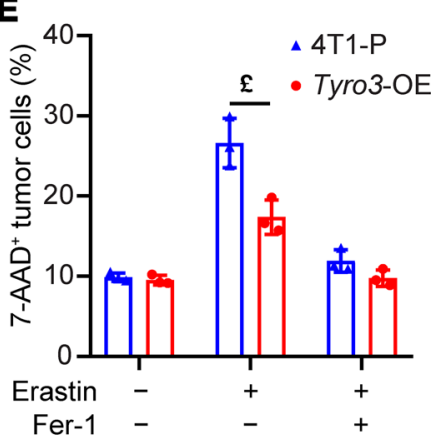

H

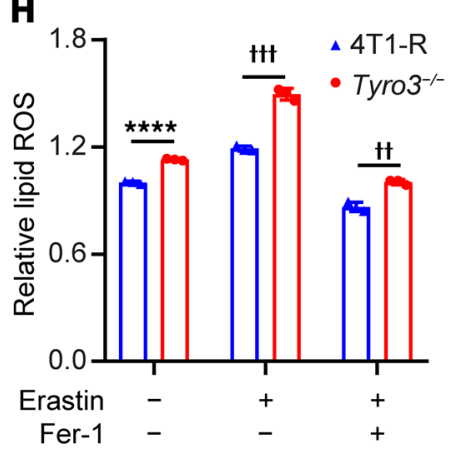

K

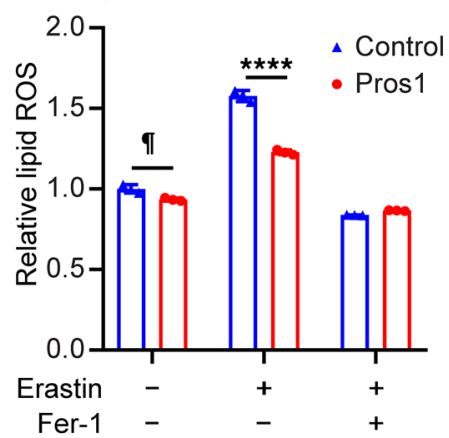

C
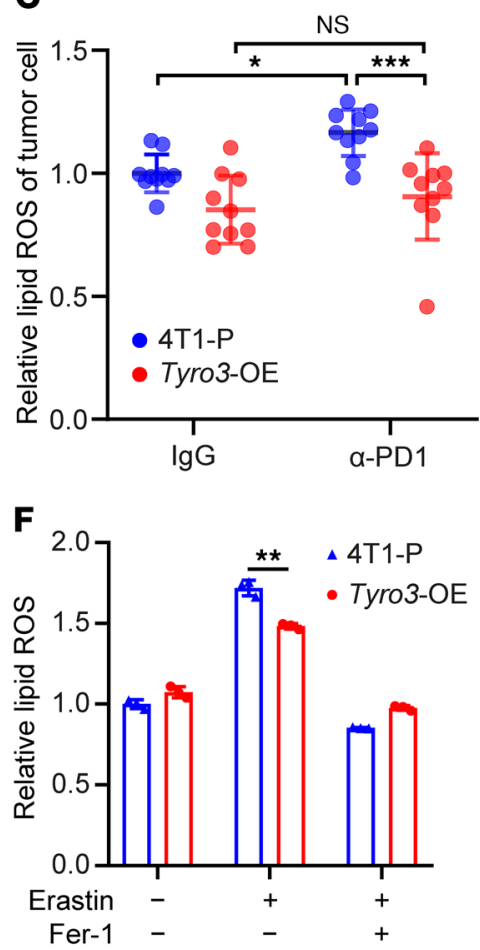

I

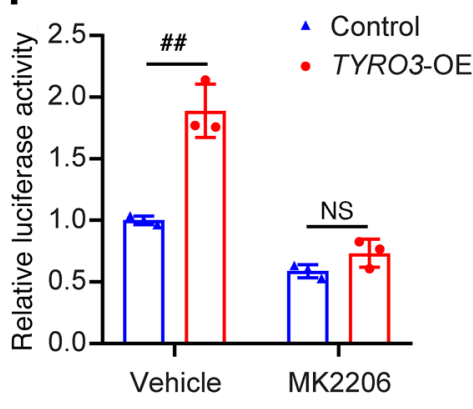

$\mathbf{L}$

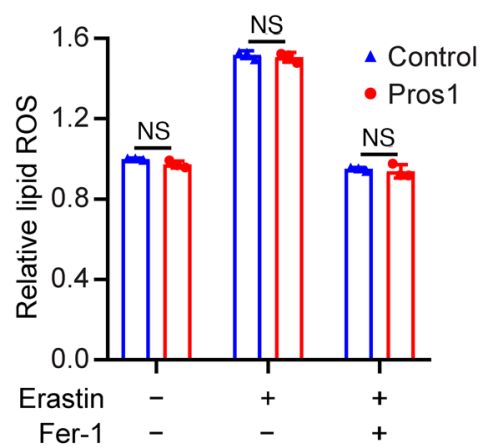


Figure 4. TYR03 suppresses tumor cell ferroptosis. (A) Volcano plot of 4T1-P and Tyro3-OE differentially expressed genes. adj, adjusted. (B) SLC3A2 expression in patients with melanoma with high $(n=52)$ and low $(n=19)$ TYRO3 expression levels who received anti-PD-1 therapy. ****P $<0.0001$, by 2-tailed, unpaired Student's $t$ test. (C) Relative lipid ROS in CD45- tumor cells. 4T1-P plus IgG versus 4T1-P plus anti-PD-1, ${ }^{*} P=0.037$; Tyro3-OE plus IgG versus Tyro3-OE plus anti-PD-1, NS $P=0.92$; and 4T1-P plus anti-PD-1 versus Tyro3-OE plus anti-PD-1, ${ }^{* *} P=0.0004$, by 2-way ANOVA. (D) MFI of IFN- $\gamma$ expression in CD8 ${ }^{+} \mathrm{T}$ cells from anti-PD-1-treated 4T1-P and Tyro3-OE tumors. NS $P=0.626$, by 2-tailed, unpaired Student's $t$ test. (E) Percentage of 7-AAD+ cells in 4T1-P and Tyro3-OE cells treated with $2 \mu \mathrm{M}$ erastin and/or $5 \mu \mathrm{M}$ Fer- 1 for 48 hours $(n=3)$. ${ }^{\mathrm{E}} P=0.013$, by 2-tailed, unpaired Student's $t$ test. (F) Relative lipid ROS in 4T1-P and Tyro3-OE cells treated with $10 \mu \mathrm{M}$ erastin and/or $10 \mu \mathrm{M}$ Fer- 1 for 8 hours $(n=3) .{ }^{*} P=0.0013$, by 2-tailed, unpaired Student's $t$ test. (G) Percentage of 7-AAD+ cells in 4T1-R and Tyro3 ${ }^{-1}$ cells treated with $2 \mu \mathrm{M}$ erastin and/ or $5 \mu \mathrm{M}$ Fer- 1 for 24 hours $(n=3) .{ }^{* * *} P<0.0001$, by 2 -tailed, unpaired Student's $t$ test. $(\mathbf{H})$ Relative lipid ROS in 4T1-R and Tyro $3^{-1 /}$ cells treated with $10 \mu \mathrm{M}$ erastin and/or $10 \mu \mathrm{M}$ Fer- 1 for 8 hours $(n=3) .{ }^{* * *} P<0.0001$, ${ }^{t+t} P=0.000124$, and ${ }^{+\dagger} P=0.00125$, by 2-tailed, unpaired Student's $t$ test. (I) A dual-luciferase reporter assay was performed by cotransfecting ARE-reporter-luciferase and pRL-TK with a TYRO3-OE plasmid, and cells were primed with $2 \mu \mathrm{M}$ MK2206 for 24 hours $(n=3)$. $\# \#=0.002$ and NS $P=0.115$, by 2-tailed, unpaired Student's $t$ test. (J) Relative lipid ROS in 4T1-P and Tyro3-OE cells primed with $2 \mu \mathrm{M}$ MK2206 for 24 hours, and then treated with $10 \mu \mathrm{M}$ erastin for 8 hours $(n=3) .{ }^{\S} P=0.02,{ }^{\S} P=0.003$, NS $P=$ 0.052 , and NS $P=0.79$, by 2-tailed, unpaired Student's $t$ test. (K) Relative lipid ROS in 4T1 cells primed with or without $200 \mathrm{nM}$ Pros1 for 24 hours and then treated with $10 \mu \mathrm{M}$ erastin and/or $10 \mu \mathrm{M}$ Fer-1 for 8 hours $(n=$ 3). " $P=0.013$ and ${ }^{* * *} P<0.0001$, by 2 -tailed, unpaired Student's $t$ test. (L) Relative lipid ROS in $4 \mathrm{~T} 1 \mathrm{TyrO}^{-/}$cells primed with or without $200 \mathrm{nM}$ Pros 1 for 24 hours and then treated with $10 \mu \mathrm{M}$ erastin and/or $10 \mu \mathrm{M}$ Fer-1 for 8 hours $(n=3)$. NS $P=0.059$, NS $P=0.53$, and NS $P=0.58$, by 2 -tailed, unpaired Student's $t$ test. Data are presented as the mean \pm SD.

respond to immunotherapy (30). Few therapeutic options are left for those patients with TNBC who develop immunotherapy resistance. Therefore, TYRO3 inhibition provides another therapeutic option for anti-PD-1/PD-L1-resistant patients with aggressive cancer types like TNBC and could result in better survival outcomes for this particular patient population.

Previous studies showed that TYRO3 is required for tumor cell proliferation $(31,32)$, and we also found that Tyro3 depletion decreased proliferation of the B16F10 melanoma cell line (our unpublished observations). However, Tyro3 overexpression or depletion had a negligible impact on TNBC cell proliferation in the current study, which is consistent with the findings of a previous report (33). Thus, TYRO3 may promote tumor cell proliferation in a cell context-dependent manner. Although the function of TYRO3 in tumor cell growth may vary among different cancer types, TYRO3 correlated well with a poor prognosis for patients with various cancer types who were receiving conventional therapies (Supplemental Figure 1D), and with therapeutic resistance to anti-PD-1/PD-L1 treatment for patients with different types of cancer (Figure 1, C, E, and H, and Supplemental Figure 1F). Our results suggested a general role of TYRO3 that is critical for cancer development and immunotherapy resistance. Future study of this resistance mechanism in other cancer models would help expand the indications for TYRO3-targeted therapies.

There is emerging evidence for the importance of TAM kinase family members in the host immune response in the TME. Two studies reported that TAM inhibition attenuates the protumor effects of myeloid-derived suppressor cells and increases $\mathrm{T}$ cell infiltration $(34,35)$. Another study showed that TAM inhibition also increases M1-polarized TAMs and activates NK cells (36). Whereas targeting TAM was the focus of these 3 studies, in the current study, we demonstrated that tumor-expressed TYRO3, and not AXL or MerTK, participated in anti-PD-1/PD-L1 resistance by suppressing tumor ferroptosis. Because TYRO3 is structurally similar to other TAM kinases, in vivo experiments to determine the specificity of TYRO3 inhibitors are needed in future studies. In addition, further development of TYRO3-specific therapeutic monoclonal antibodies could improve the efficacy of immunotherapy by minimizing off-target effects.

In summary, our findings reveal that TYRO3 promoted tumor cell resistance to anti-PD-1/PD-L1 therapy by inhibiting ferroptosis and supporting a protumor TME and provide fundamental insight into how the RTK signaling axis impairs antitumor immunity by perturbing the cell death pathways and remodeling the TME. TYRO3 is, therefore, a potential therapeutic target for overcoming resistance in this setting. Moreover, our results also suggest a role for TYRO3 as a predictive biomarker for patient stratification to improve therapeutic efficacy, especially for those patients whose tumors do not respond to anti-PD-1/PD-L1 therapy.

\section{Methods}

Cell lines and culture. The following cell lines were obtained from the American Type Culture Collection (ATCC): 4T1 (murine mammary tumor cells), 293T (human kidney cells), B16F10 (murine melanoma cells), BT549 (human breast cancer cells), THP1 (human acute monocytic leukemia cells), and PY8119 (murine mammary tumor cells). Cells were validated by short tandem repeat DNA fingerprinting at The University of Texas MD Anderson Cancer Center and confirmed to be negative for mycoplasma contamination. 4T1, 293T, B16F10, and PY8119 cells were cultured in DMEM (SH3024301, Cytiva HyClone) with 10\% FBS (10437028, Gibco, Thermo Fisher Scientific). BT549 and THP1 cells were cultured in RPMI 1640 media (RPL09, Caisson Laboratories) with 10\% FBS.

BMDMs were isolated from BALB/c mouse femur bone. A singlecell suspension was prepared and cultured in DMEM media with 10 ng/mL mouse macrophage CSF (576402, BioLegend) for 7 days, and then cultured in CM for 24 hours with the addition of $50 \mathrm{ng} / \mathrm{mL}$ mouse IFN- $\gamma$ (575304, BioLegend) and $100 \mathrm{ng} / \mathrm{mL}$ LPS (L6529, MilliporeSigma) for M1 macrophage polarization, or with $10 \mathrm{ng} / \mathrm{mL}$ mouse IL-4 (NBP2-35131, Novus Biologicals) for M2 macrophage polarization. In the THP1 monocyte polarization experiment, cells were differentiated into macrophages with $200 \mathrm{nM}$ PMA (P8139, MilliporeSigma) for 24 hours, and then cultured with CM from BT549 parental cells and BT549 Tyro3-OE cells for 24 hours, in the presence or absence of axitinib ( $5 \mu \mathrm{M}, \mathrm{S1005}$, Selleck Chemical), with the addition of $50 \mathrm{ng} /$ $\mathrm{mL}$ human IFN- $\gamma$ (570202, BioLegend) and $100 \mathrm{ng} / \mathrm{mL}$ LPS for M1 macrophage polarization or $20 \mathrm{ng} / \mathrm{mL}$ human IL-4 (200-04, Peprotech) for M2 macrophage polarization.

Tyro3-OE 4T1 cells were established by lentiviral infection of 4T1-P cells using the Tyro3-pCDH, pMD2.G, and pSPAX2 lentiviral packaging system. Tyro3-KO cells (Tyro3--) were established by transfecting 4T1-R cells using a CRISPR/Cas9 Double Nickase Plasmid (sc-423567-NIC, Santa Cruz Biotechnology). 
A

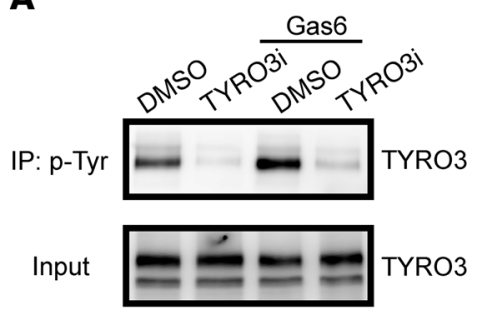

B

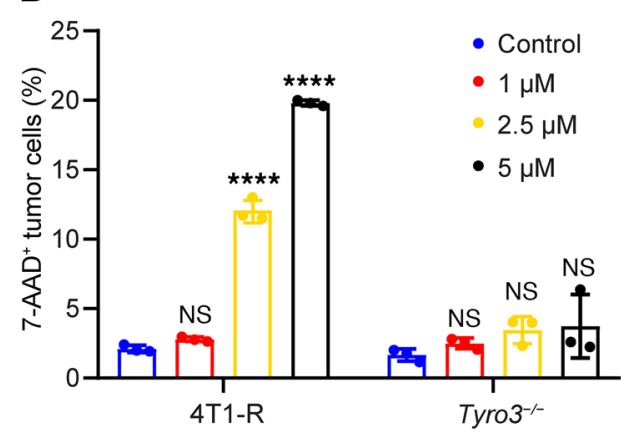

C

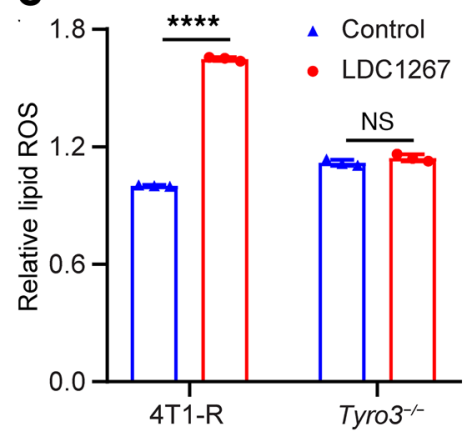

D

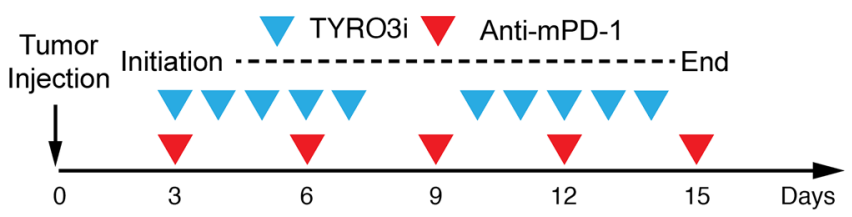

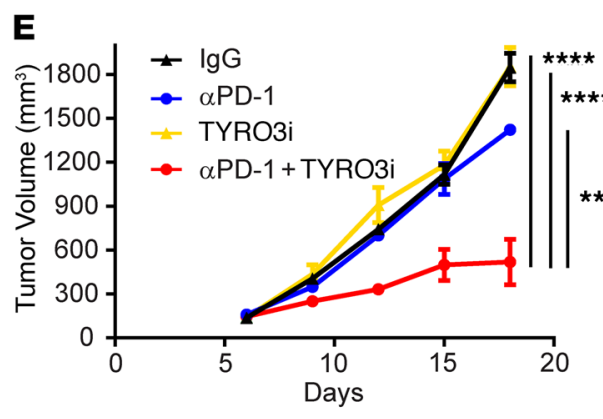

G

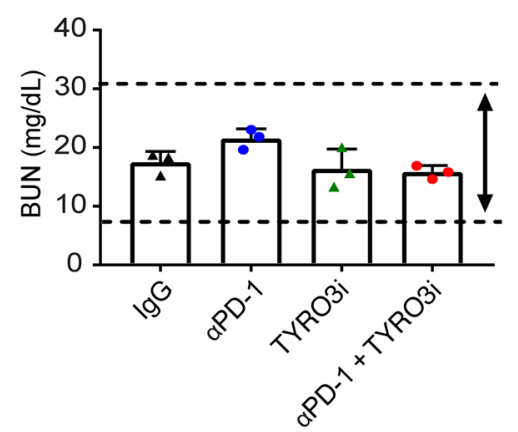

\section{F}

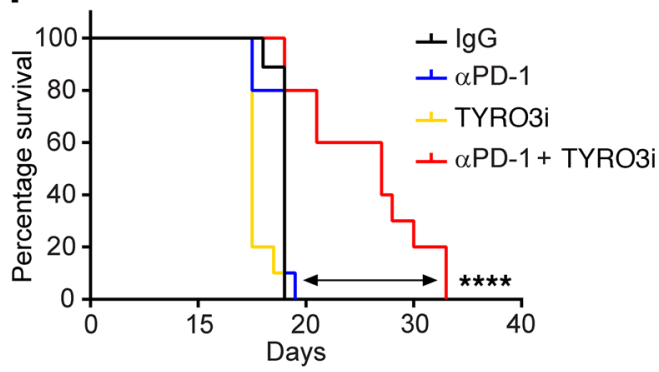

I

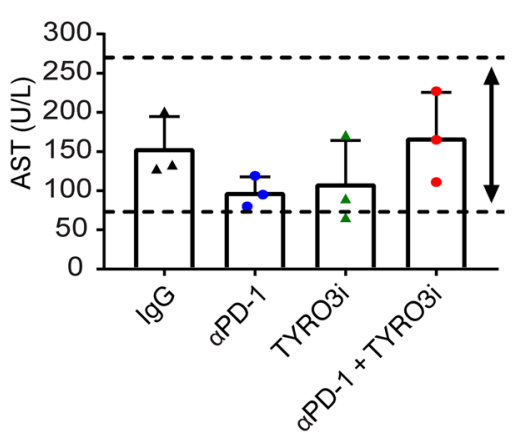

J

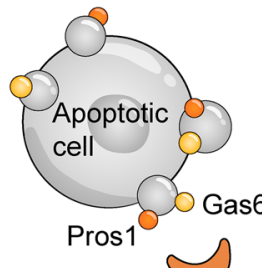

TYRO3

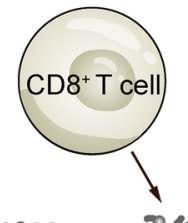

ஃ VEGF

○ Gas6

O Pros1

Apoptotic bodies
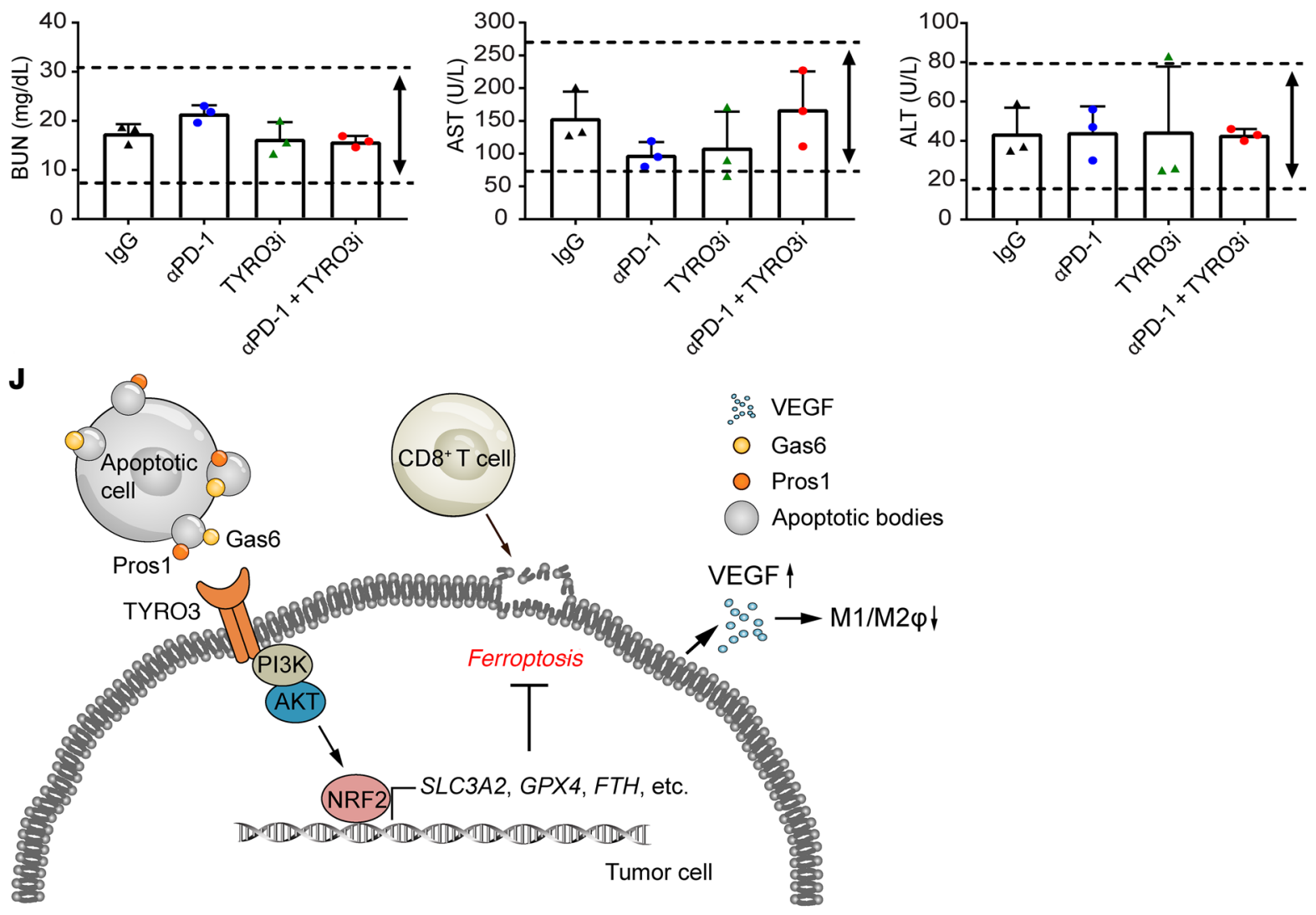
Figure 5. Inhibition of TYR03 enhances ferroptosis and sensitizes resistant tumors to anti-mPD-1 therapy. (A) Immunoprecipitation followed by Western blot analysis of TYRO3 tyrosine phosphorylation ( $p$-Tyr) in 4T1 cells treated with the TYRO3 inhibitor (TYRO3i) LDC1267 ( $2.5 \mu \mathrm{M}, 2.5$ hours) or control (DMSO), and in the presence or absence of Gas6 (100 nM, 30 minutes). TYRO3 served as a loading control.. (B) Percentage of 7-AAD+ cells in 4T1-R or Tyro $3^{-1-}$ cells treated with $0,1,2.5$, and $5 \mu$ M LDC1267 for 24 hours $(n=3)$. NS $P=0.94,{ }^{* * * *} P<0.0001$, NS $P=0.87$, NS $P=0.19$, and NS $P=0.09$, by 2-way ANOVA. (C) Relative lipid ROS in 4T1-R or Tyro3 $1-$ cells treated with $5 \mu \mathrm{M}$ LDC1267 for 12 hours $(n=3) .{ }^{* * *} P<0.0001$ and NS $P$ $=0.16$, by 2-tailed, unpaired Student's $t$ test. (D) Schematic showing the treatment schedule to evaluate the combination of LDC1267 and anti$\mathrm{mPD}-1$ treatment in mice. LDC1267 was intraperitoneally injected into mice starting on the third day after tumor inoculation for a total of 2 rounds, with 5 treatments for each round. Anti-mPD-1 was intraperitoneally injected into mice for a total of 5 treatments. (E) Growth of 4T1-R tumors in mice that were given anti-mPD-1, LDC1267, or their combination. IgC treatment served as a control ( $n=10$ mice per group). ${ }^{* * *} P<0.0001$ and ${ }^{*} P=0.0039$, by 2 -way ANOVA. Data are presented as the mean \pm SEM. (F) Survival of mice in each group. ${ }^{* * *} P<0.0001$, by 2 -sided log-rank test (TYRO3i plus anti-PD-1 versus anti-PD-1 alone). (C) Indicators of liver and kidney function in mice. The normal range for BUN, (H) AST, and (I) ALT are indicated by the dashed lines ( $n=3$ mice per group). (J) Schematic of the proposed model showing that TYRO3 inhibits tumor ferroptosis and supports a protumor TME by reducing the ratio of M1/M2 macrophages, thus promoting anti-PD-1 therapy resistance.

Animal studies and establishment of resistance models. Six-week-old BALB/c mice were purchased from The Jackson Laboratory. Mouse $4 \mathrm{~T} 1$ cells $\left(5 \times 10^{4}\right.$ cells) in $50 \mu \mathrm{L}$ of $50 \%$ Matrigel (47743-720, Corning) were injected into the mammary fat pad. Three days after inoculation, $100 \mu \mathrm{g}$ mouse anti-PD-1 antibody (BE0146, Bio X Cell) or IgG control (BE0089, Bio X Cell) was injected intraperitoneally twice a week for a total of 5 injections. For the TYRO3 inhibitor and anti-mPD-1 combination treatment, mice were also treated daily with vehicle control (90\% polyethylene glycol 400 and 10\% DMSO) or LDC1267 (20 mg/ $\mathrm{kg}$, S7638, Selleck Chemical) for 10 days by intraperitoneal injection. Tumor volume was measured using the formula: length $\times$ width $^{2} / 2$, where length is the longest diameter of the tumor and width is the shortest diameter. Survival analysis was performed using GraphPad Prism 8 (GraphPad Software). A mouse tumor volume of $1500 \mathrm{~mm}^{3}$ was set as the endpoint.

For the in vivo anti-PD-1 resistant model, 4T1-Luc2 mouse tumor cells expressing luciferase $\left(5 \times 10^{4}\right.$ cells $)$ were inoculated into the mammary fat pad of BALB/c mice. Anti-mPD- 1 was administered to the mice 4 times at 3-day intervals. Tumors were harvested after they reached the endpoint size and were dissociated for culturing. The treatment cycle was repeated 2 more times to obtain 4T1-R cells.

Western blotting and immunoprecipitation. Cell lysates were prepared in lysis buffer containing $50 \mathrm{mM}$ Tris- $\mathrm{HCl}(\mathrm{pH} 7.4), 150 \mathrm{mM}$ $\mathrm{NaCl}, 0.1 \%$ sodium dodecyl sulfate, $1 \%$ Triton $\mathrm{X}-100,1 \times$ phosphatase inhibitor cocktail (B15002, Bimake), and 1× protease inhibitor cocktail (B14001, Bimake). Protein samples were separated by SDS-PAGE, transferred onto a PVDF membrane, and subjected to Western blot analysis using the indicated antibodies. For immunoprecipitation, cell lysates were prepared in lysis buffer containing $50 \mathrm{mM}$ Tris- $\mathrm{HCl}(\mathrm{pH}$ 7.4), $150 \mathrm{mM} \mathrm{NaCl}, 1 \%$ Triton X-100, $1 \times$ phosphatase inhibitor cocktail, and $1 \times$ protease inhibitor cocktail. Cell lysates $(2 \mathrm{mg})$ from each group were incubated with $4 \mu \mathrm{g}$ anti-p-tyrosine antibody overnight at $4^{\circ} \mathrm{C}$, followed by the addition of $20 \mu \mathrm{L} 50 \%$ BSA preblocking protein A mag- netic beads (1614013, Bio-Rad) for 1 hour at $4^{\circ} \mathrm{C}$. Beads were washed 3 times with lysis buffer and then subjected to immunoblotting. The following antibodies were used for Western blotting and immunoprecipitation: TYRO3 (5585S, Cell Signaling Technology), tubulin (T5168, MilliporeSigma), and p-tyrosine (05-321, EMD Millipore).

IHC staining. Tumor specimens from patients with lung cancer who received anti-PD-1 or anti-PD-L1 antibody therapy $(n=29)$ were obtained from Harbin Medical University. Briefly, tumor specimens were incubated with an antibody against TYRO3 (1:100; OM223993, Omnimabs) or p-TYRO3 (1:100; PAB29209, Abnova) and then treated with a biotinylated secondary antibody followed by incubation with an avidin-biotin-peroxidase complex solution. Immunoreactivity was visualized using DAB. The protein expression score was calculated from both the percentage $(0 \%-100 \%)$ of cells stained positive and the immunostaining intensity ( 0 , negative; 1 , weak; 2 , moderate; 3 , strong). Paraffin-embedded tissue array slides containing melanoma sections (ME804b, US Biomax) were pretreated using a Melanin Bleach Kit (24883-1, Polysciences) and stained with anti-SLC3A2 antibody (1:100; 47213S, Cell Signaling Technology) and anti-TYRO3 (1:100; OM223993, Omnimabs) as described above. Slides were visualized using an amino ethyl carbazole chromogen (Supplemental Figure $4 \mathrm{C}$ ). The intensity of staining was assigned to 1 of 2 groups according to the histologic scores: high and medium (+++/++) and negative and low $(-/+)$.

Two pathologists were tasked with independently evaluating the IHC results using an established semiquantitative approach to determine the $\mathrm{H}$ score.

Real-time quantitative PCR. Total RNA products were extracted from the indicated cells using an RNA Extraction Kit (50-444-622, Zymo Research) and converted to cDNA with a Reverse Transcription Kit (4368814, Thermo Fisher Scientific). Real-time PCR reaction mix was prepared by mixing cDNA, primers, and $2 \times$ SYBR green master mix (1708884, Bio-Rad) and adding $\mathrm{H}_{2} \mathrm{O}$ to the $20 \mu \mathrm{L}$ volume. The reaction mix was run according to the following protocol: denaturation at $95^{\circ} \mathrm{C}$ for 3 minutes; amplification $\left(45\right.$ cycles) at $95^{\circ} \mathrm{C}$ for 10 seconds, $60^{\circ} \mathrm{C}$ for 10 seconds, and $72^{\circ} \mathrm{C}$ for 30 seconds. The sequences of the primers used for real-time quantitative PCR are listed in Supplemental Table 2.

RNA-Seq and data analysis. Total RNA was extracted from the indicated cells and subjected to whole-transcriptome analysis according to the standard procedure provided by Applied Biosystems. Hierarchical clustering analysis was carried out with the $\log _{10}$ (fragments per kilobase per million mapped reads +1 [FPKM +1$]$ ) of genes that were differentially expressed in all comparison groups. RNA-Seq data were deposited in the NCBI's Gene Expression Omnibus (GEO) repository (GEO GSE167467).

CyTOF and data analysis. Tumors harvested from mice were digested using the Tumor Dissociation Kit (130-096-730, Miltenyi Biotec). Prior to staining with CyTOF antibodies, the cells were incubated with anti-mouse CD16/CD32 antibody (101319, BioLegend) for 15 minutes to block Fc receptors. The cells were then incubated with a metal-labeled monoclonal antibody cocktail. After permeabilization by methanol, the cells were incubated with intracellular antibodies. The samples were washed and diluted in double-distilled $\mathrm{H}_{2} \mathrm{O}$ containing bead standards, followed by mass cytometric analysis. All mass cytometry profiles were normalized and manually gated using FlowJo software according to DNA, event length, live/dead discrimination, CD45, 
and 4 bead channels to exclude dead cells, debris, doublets, and nonimmune cells and beads. Unsupervised PhenoGraph clustering analysis in the cytofkit package in $\mathrm{R}$ was performed to identify immune profiles automatically. Heatmaps were generated according to the mean value for each marker in all clusters (Supplemental Figure 3A).

Flow cytometry. For M1 and M2 macrophage marker measurement, BMDMs were trypsinized and stained with fixable viability dyes for 15 minutes, and then the cells were washed and stained with antiCD16/CD32 followed by incubation with the indicated antibodies (F4/80, CD11c, and CD206) for 1 hour and analyzed by flow cytometry. All data were normalized to the control samples and are shown as the relative mean fluorescence intensity (MFI) of CD11c or CD206.

For BODIPY-C11 staining, tumor cells $\left(1 \times 10^{5}\right.$ cells/well $)$ were seeded in 6-well plates and treated with drugs for the indicated durations, and the cells were then stained with DMEM culture containing $5 \mu \mathrm{M}$ BODIPY 581/591 C11 and incubated for 30 minutes at $37^{\circ} \mathrm{C}$ in a cell culture incubator. Cells were washed twice using PBS and digested by trypsin followed by DMEM neutralization. Samples were analyzed immediately by flow cytometer, and the signals from both nonoxidized C11 (phycoerythrin [PE] channel) and oxidized C11 (FITC channel) were monitored. The MFI of FITC was calculated. All data were normalized to the control samples and are shown as relative lipid ROS.

To evaluate the lipid peroxidation in tumor samples from animals that received anti-PD-1 therapy, 4T1 tumors were collected and disassociated. A small fraction of the single-cell pellet was suspended in red blood cell lysis buffer for 3 minutes on ice and then washed and stained with anti-CD16/CD32 for 15 minutes, followed by incubation with an anti-CD45 antibody. Next, cells were stained with BODIPY 581/591 C11 for 30 minutes. The tumor suspension was washed using PBS and analyzed immediately by flow cytometer.

For cell death analysis, the cells were treated, collected, and suspended in PBS containing $1 \mu \mathrm{g} / \mathrm{mL}$ 7-aminoactinomycin D for $5 \mathrm{~min}$ utes and then directly run on a flow cytometer.

The following antibodies or chemicals were used in this study: antimouse CD16/CD32 (101319, BioLegend); FITC anti-mouse F4/80 (123107, BioLegend); PE/Cy7 anti-mouse CD11c (117317, BioLegend); APC anti-mouse CD206 (141707, BioLegend); APC anti-mouse CD45 (103112, BioLegend); erastin (17754, Cayman Chemicals); ferrostatin 1 (17729, Cayman Chemicals); MK-2206 (11593, Cayman Chemicals), and BODIPY 581/591 C11 (D3861, Thermo Fisher Scientific).

MDA assay. MDA content in tumor cells was measured using the Lipid Peroxidation MDA Assay Kit (ab118970, Abcam). In this assay, MDA in the cells reacts with thiobarbituric acid (TBA) and generates an MDA-TBA adduct, which can be quantified fluorometrically. A total of $2 \times 10^{6}$ cells were collected and homogenized in $300 \mu \mathrm{L}$ MDA lysis buffer, and the cell lysate was sonicated and centrifuged to remove insoluble material. A total of $200 \mu \mathrm{L}$ supernatant was mixed with 600 $\mu \mathrm{L}$ TBA solution and incubated at $95^{\circ} \mathrm{C}$ for 1 hour. The MDA-TBA complex concentration was measured on a microplate reader with rel- ative fluorescence units at $\mathrm{Ex} / \mathrm{Em}=532 / 553 \mathrm{~nm}$ or $\mathrm{OD}=532 \mathrm{~nm}$ and was normalized to the protein concentration.

Statistics. Statistical analyses were performed using GraphPad Prism software. All data are presented as the mean \pm SD unless otherwise noted. A 2-tailed Student's $t$ test was used to compare 2 groups of independent samples unless otherwise noted. ANOVA, 2-tailed unless otherwise noted, was used to determine the variation among or between groups. A $P$ value of less than 0.05 was considered statistically significant.

Study approval. All animal experiments were performed under guidelines approved by IACUC of The University of Texas MD Anderson Cancer Center. Under IRB approval of The Affiliated Tumor Hospital of Harbin Medical University and The University of Texas MD Anderson Cancer Center, a total of 29 diagnosed patients with a pathologically and/or radiologically confirmed lung cancer diagnosis were recruited during 2016-18 as part of an ongoing study. Written informed consent was obtained from all patients. The tissue samples were collected before immunotherapy.

\section{Author contributions}

$\mathrm{MCH}$ supervised the study. ZJ, SOL, HY, and MCH designed the experiments. SOL established the mouse resistant cell lines and Tyro3 $^{-/-} 4 \mathrm{~T} 1$ cells. SOL and SSC performed RTK screening assays. MY and YW performed immunochemical staining experiments. JY analyzed the database of patients with melanoma. BK performed the toxicity test, and ZJ performed the remaining experiments. HHL, JMH, LCC, GNH, CL, LY, and DY provided scientific input. ZJ, MCH, and JLH wrote the manuscript.

\section{Acknowledgments}

We thank Chia-Wei Li, Jong-Ho Cha, and Jielin Liu for insightful discussions and suggestions. We thank Weiya Xia for her assistance with IHC experiments. We also thank Sarah Bronson from Scientific Publications at The University of Texas, MD Anderson Cancer Center for manuscript editing. Funding for this work was provided by a 2019 AACR-Pfizer Immuno-oncology Research Fellowship (19-40-49-JIAN); the Breast Cancer Research Foundation (BCRF-19-070); an MD Anderson National Cancer Institute Cancer Center support grant, National Cancer Institute (NCI) (CCSG CA016672); the Cancer Prevention and Research Institute of Texas (RP160710, MIRA grant RP160710); the National Breast Cancer Foundation Inc.; the Patel Memorial Breast Cancer Endowment Fund; The University of Texas MD Anderson-China Medical University and Hospital Sister Institution Network Fund; and the Center for Biological Pathways.

Address correspondence to: Mien-Chie Hung, China Medical University, 100, Sec 1 Jingmao Rd, Beitun District, Taichung 40406, Taiwan. Phone: 886.04.22057153; Email: mhung@ cmu.edu.tw.
1. Hamid O, et al. Five-year survival outcomes for patients with advanced melanoma treated with pembrolizumab in KEYNOTE-001. Ann Oncol. 2019;30(4):582-588.

2. Garon EB, et al. Pembrolizumab for the treatment of non-small-cell lung cancer. $N$ Engl J Med. 2015;372(21):2018-2028.
3. Adams S, et al. Pembrolizumab monotherapy for previously untreated, PD-L1-positive, metastatic triple-negative breast cancer: cohort B of the phase II KEYNOTE-086 study. Ann Oncol. 2019;30(3):405-411.

4. El-Khoueiry AB, et al. Nivolumab in patients with advanced hepatocellular carcinoma (CheckMate
040): an open-label, non-comparative, phase $1 / 2$ dose escalation and expansion trial. Lancet. 2017;389(10088):2492-2502.

5. Hirano F, et al. Blockade of B7-H1 and PD-1 by monoclonal antibodies potentiates cancer therapeutic immunity. Cancer Res. 2005;65(3):1089-1096. 
6. Regad T. Targeting RTK signaling pathways in cancer. Cancers (Basel). 2015;7(3):1758-1784.

7. Smart SK, et al. The emerging role of TYRO3 as a therapeutic target in cancer. Cancers (Basel). 2018;10(12):E474.

8. Wang $\mathrm{W}$, et al. $\mathrm{CD} 8^{+} \mathrm{T}$ cells regulate tumour ferroptosis during cancer immunotherapy. Nature. 2019;569(7755):270-274.

9. Li CW, et al. Eradication of triple-negative breast cancer cells by targeting glycosylated PD-L1. Cancer Cell. 2018;33(2):187-201.

10. Jiang $P$, et al. Signatures of $T$ cell dysfunction and exclusion predict cancer immunotherapy response. Nat Med. 2018;24(10):1550-1558.

11. Gentles AJ, et al. The prognostic landscape of genes and infiltrating immune cells across human cancers. Nat Med. 2015;21(8):938-945.

12. Riaz N, et al. Tumor and microenvironment evolution during immunotherapy with nivolumab. Cell. 2017;171(4):934-949.

13. Graham DK, et al. The TAM family: phosphatidylserine sensing receptor tyrosine kinases gone awry in cancer. Nat Rev Cancer. 2014;14(12):769-785.

14. Reissfelder C, et al. Tumor-specific cytotoxic T lymphocyte activity determines colorectal cancer patient prognosis. JClin Invest. 2015;125(2):739-751.

15. Linger RM, et al. TAM receptor tyrosine kinases: biologic functions, signaling, and potential therapeutic targeting in human cancer. Adv Cancer Res. 2008;100:35-83.

16. Cancer Genome Atlas Network. Genomic classification of cutaneous melanoma. Cell. 2015;161(7):1681-1696.
17. Vajaitu C, et al. The central role of inflammation associated with checkpoint inhibitor treatments. JImmunol Res. 2018;2018:4625472.

18. Wen Q, et al. The release and activity of HMGB1 in ferroptosis. Biochem Biophys Res Commun. 2019;510(2):278-283.

19. Melchiotti R, et al. Cluster stability in the analysis of mass cytometry data. Cytometry $A$. 2017;91(1):73-84.

20. Kloepper J, et al. Ang-2/VEGF bispecific antibody reprograms macrophages and resident microglia to anti-tumor phenotype and prolongs glioblastoma survival. Proc Natl Acad Sci U S A. 2016;113(16):4476-4481.

21. Hao S, et al. Metabolic networks in ferroptosis. Oncol Lett. 2018;15(4):5405-5411.

22. Abdalkader M, et al. Targeting Nrf2 to suppress ferroptosis and mitochondrial dysfunction in neurodegeneration. Front Neurosci. 2018;12:466.

23. Xie Y, et al. Ferroptosis: process and function. Cell Death Differ. 2016;23(3):369-379.

24. Sun X, et al. Activation of the p62-Keap1-NRF2 pathway protects against ferroptosis in hepatocellular carcinoma cells. Hepatology. 2016;63(1):173-184.

25. Dodson M, et al. NRF2 plays a critical role in mitigating lipid peroxidation and ferroptosis. Redox Biol. 2019;101107.

26. Martin D, et al. Regulation of heme oxygenase-1 expression through the phosphatidylinositol 3-kinase/Akt pathway and the Nrf2 transcription factor in response to the antioxidant phytochemical carnosol. J Biol Chem. 2004;279(10):8919-8929.

27. Tsou WI, et al. Receptor tyrosine kinases, TYRO3, AXL, and MER, demonstrate distinct patterns and complex regulation of ligand-induced activa- tion. J Biol Chem. 2014;289(37):25750-25763.

28. Paolino M, et al. The E3 ligase Cbl-b and TAM receptors regulate cancer metastasis via natural killer cells. Nature. 2014;507(7493):508-512.

29. Liedtke C, et al. Response to neoadjuvant therapy and long-term survival in patients with triple-negative breast cancer. JClin Oncol. 2008;26(8):1275-1281.

30. Mavratzas A, et al. Atezolizumab for use in PD-L1-positive unresectable, locally advanced or metastatic triple-negative breast cancer. Future Oncol. 2020;16(3):4439-4453.

31. Brown JE, et al. Cross-phosphorylation, signaling and proliferative functions of the Tyro3 and Axl receptors in Rat 2 cells. PLoS One. 2012;7(5):e36800.

32. Chien CW, et al. Targeting TYRO3 inhibits epithelial-mesenchymal transition and increases drug sensitivity in colon cancer. Oncogene. 2016;35(45):5872-5881.

33. Ekyalongo RC, et al. TYRO3 as a potential therapeutic target in breast cancer. Anticancer Res. 2014;34(7):3337-3345.

34. Holtzhausen A, et al. TAM family receptor kinase inhibition reverses MDSC-mediated suppression and augments anti-PD-1 therapy in melanoma. Cancer Immunol Res. 2019;7(10):1672-1686.

35. Kasikara C, et al. Pan-TAM tyrosine kinase inhibitor BMS-777607 enhances anti-PD-1 mAb efficacy in a murine model of triple-negative breast cancer. Cancer Res. 2019;79(10):2669-2683.

36. Yokoyama $Y$, et al. Immuno-oncological efficacy of RXDX-106, a novel TAM (TYRO3, AXL, MER) family small-molecule kinase inhibitor. Cancer Res. 2019;79(8):1996-2008. 